\title{
MANUAL “TODA HORA É HORA DE CUIDAR" E E-BOOK CONCEITUAL: PROMOÇÃO DA SAÚDE REVISITADA
}

\section{Alfredo Almeida Pina-Oliveira}

Enfermeiro e Educador em Saúde Pública. Docente Doutor do Programa Stricto sensu de Enfermagem da Universidade Guarulhos (UNG) e do Centro Universitário Campo Limpo Paulista (UNIFACCAMP), São Paulo (SP), Brasil

\section{Anna Maria Chiesa}

Enfermeira. Livre docente em Enfermagem. Docente de Coletiva da Escola de Enfermagem da Universidade de São Paulo (USP), São Paulo (SP), Brasil. Associada do Departamento de Enfermagem em Saú-

RESUMO: Este estudo teve como objetivo analisar as dimensões de saúde e bem-estar contidas em materiais educativos impressos que enfatizam a promoção da saúde na primeira infância. Pesquisa qualitativa sobre a avaliação crítica de manual e e-book conceitual relacionado à promoção da saúde na primeira infância baseada no referencial de saúde e bem-estar de Ronald Labonte. Evidenciou-se por meio da frequência temática a ênfase da dimensão biológica nos documentos analisados, porém com a incorporação transversal das dimensões afetivas e sociais associadas a abordagens críticas para o cuidado de crianças, sem desconsiderar suas famílias e suas comunidades. $\mathrm{O}$ diagrama de Labonte permitiu avaliar de modo dialético os conteúdos de promoção da saúde em materiais instrucionais elaborados para a educação de profissionais que promovem a saúde e o bem-estar infantil.

PALAVRAS-CHAVE: Manuais como assunto; Promoção da saúde; Desenvolvimento infantil; Capacitação em serviço; Alfabetização em saúde.

\section{HANDBOOK "TODA HORA É HORA DE CUIDAR" AND CONCEPTUAL E-BOOK: REVISITING HEALTH PROMOTION}

\begin{abstract}
Current paper analyzes health and well-being in educational printed materials which underscore health promotion in childhood. A qualitative research on the critical evaluation of the handbook and conceptual e-book related to health promotion in childhood based on Ronald Labonte's health and well-being references. Through thematic frequency, results reveal emphasis on the biological dimension in the documents analyzed, with the transversal incorporation of affective and social dimensions, associated with critical stances for child care, without disregarding their families and communities. Labonte diagram evaluated dialectically the contents of health promotion in instructional material prepared for the education of professional who enhance health and well-being
\end{abstract}

KEY WORDS: Manuals as topic; Health promotion; Child development; Inservice training; Health literacy.

\section{INTRODUÇÃO}

Autor correspondente:

Alfredo Almeida Pina-Oliveira

E-mail: alfredo.almeida@prof.ung.br
Sintetizar evidências científicas em materiais educativos impressos (MEI) representa um desafio para as práticas educativas que 
promovem a saúde e a qualidade de vida da população e para a educação permanente de diferentes profissionais que atuam direta ou indiretamente nos diferentes níveis dos sistemas de saúde.

Manuais, revistas médicas, diretrizes de prática clínica, cadernos, cartilhas e folhetos proporcionam discreta melhoria da aprendizagem dos profissionais de saúde no tocante ao aumento da consciência, conhecimento e atitudes relacionados aos cuidados em saúde; apresentam vantagens em sua disseminação que ocorre de modo amplo e com baixo custo; porém, costumam ser utilizados de forma passiva em serviços de saúde ${ }^{1}$.

A Carta de Alfabetização em Saúde de Calgary (Canadá) preconiza diferentes estratégias para favorecer a comunicação entre os profissionais e os usuários dos sistemas de saúde, sendo que a avaliação contínua do teor dos MEI configura um dos aspectos centrais para a melhoria da qualidade e da segurança na assistência ${ }^{2}$.

Estudos avaliativos dos conteúdos de MEI foram encontrados na área de promoção da saúde da criança ${ }^{3,4}$, no cuidado em pré-natal ${ }^{5}$, no planejamento familiar ${ }^{6}$, no rastreamento da acuidade visual em escolares ${ }^{7}$, no autocuidado em portadores de diabetes $^{8}$, na abordagem de mulheres com câncer de mama ${ }^{9} \mathrm{e}$ na alta hospitalar de pessoas em oxigenoterapia prolongada ${ }^{10}$.

Os modelos avaliativos adotados para validar esses recursos instrucionais contemplam domínios da estrutura do material, dos aspectos psicoeducativos, da organização do conteúdo e do tipo de comunicação empregado $^{6,11,12}$. Nesse sentido, identifica-se a exiguidade de avaliações que primam pela compreensão dos pressupostos teóricos que fundamentam a elaboração dos MEI com ênfase no paradigma da promoção da saúde.

Destarte, os MEI são instrumentos relevantes para que os sujeitos partícipes da educação permanente realizem processos reflexivos em prol de mudanças nos serviços de saúde ${ }^{13}$, nas relações com os usuários de saúde ${ }^{5}$ e nas atividades realizadas pelo corpo docente e discente integrados a diferentes instituições de atenção de saúde ${ }^{7,8}$.

Quando aliados à experiência dos profissionais e pertinentes às discussões sobre as necessidades dos usuários dos serviços de saúde, os MEI podem subsidiar processos educacionais mais significativos cujos efeitos apresentam potencial para incrementar a equidade, a segurança e a efetividade das ações programáticas com ênfase na educação em saúde $e^{3,6,8-10}$. Sendo assim, o objetivo do presente estudo foi analisar as dimensões de saúde e bem-estar contidas em materiais educativos impressos que enfatizam a promoção da saúde na primeira infância.

\section{METODOLOGIA}

Tratou-se de uma pesquisa qualitativa, descritiva e exploratória, a partir da técnica de análise documental do manual de apoio "Toda hora é hora de cuidar" (doravante referido como manual de apoio) e do e-book conceitual do Projeto "Nossas crianças: janelas de oportunidades" (para simplificar, Projeto Janelas) $^{13,14}$.

Esses dois MEI foram elaborados de maneira interdisciplinar e intersetorial e pautados nos princípios norteadores da promoção da saúde para a fundamentação teórico-operacional da aplicação de outras duas tecnologias de cuidado para o desenvolvimento infantil saudável: a ficha de acompanhamento da criança e a cartilha da família "Toda hora é hora de cuidar"3,4,14-16.

O manual de apoio, o $e$-book conceitual, a cartilha da família e a ficha de acompanhamento da criança representam tecnologias de cuidado inovadoras para a promoção da saúde das crianças ${ }^{14}$ e estão disponíveis na íntegra em versões digitalizadas no endereço eletrônico do grupo de pesquisa "Modelos tecnoassistenciais e a promoção da saúde", da Escola de Enfermagem da Universidade de São Paulo, a saber: http://www.ee.usp. br/pesquisa/grupromo/producao.asp.

Justifica-se a avaliação do manual de apoio e do e-book conceitual por se tratarem de MEI com conteúdos inovadores sobre o desenvolvimento infantil com foco no fortalecimento das competências dos profissionais de equipes da Estratégia Saúde da Família (ESF) a fim de promover a saúde e o bem-estar de crianças de zero a seis anos, sem desconsiderar suas famílias e seus recursos comunitários $^{13-16}$.

Elegeu-se a análise temática de conteúdo como forma de "descobrir os núcleos de sentido que 
compõem uma comunicação cuja presença ou frequência signifiquem alguma coisa para o objetivo analítico visado" ${ }^{17}$ e que foi associada ao referencial teórico de Ronald Labonte ${ }^{18}$, escolhido a priori, para avaliar 0 alinhamento conceitual do manual de apoio a um modelo que contempla a dialética do conceito positivo e ampliado de saúde e avalia a dinamicidade do processo saúdedoença que deve embasar a construção de tecnologias de cuidado inovadoras com foco no desenvolvimento infantil saudável ${ }^{14}$.

O diagrama ou holosfera de saúde (Figura 1) derivou de pesquisa sobre experiências comunitárias a respeito dos conceitos relacionados à positividade da saúde $^{18}$. Essa matriz analítica apresenta três dimensões da saúde individual e três intersecções que representam o poder que o indivíduo detém para controlar e alterar os determinantes de sua saúde em um determinado momento histórico com base em um contexto macroestrutural da sociedade ${ }^{14}$.

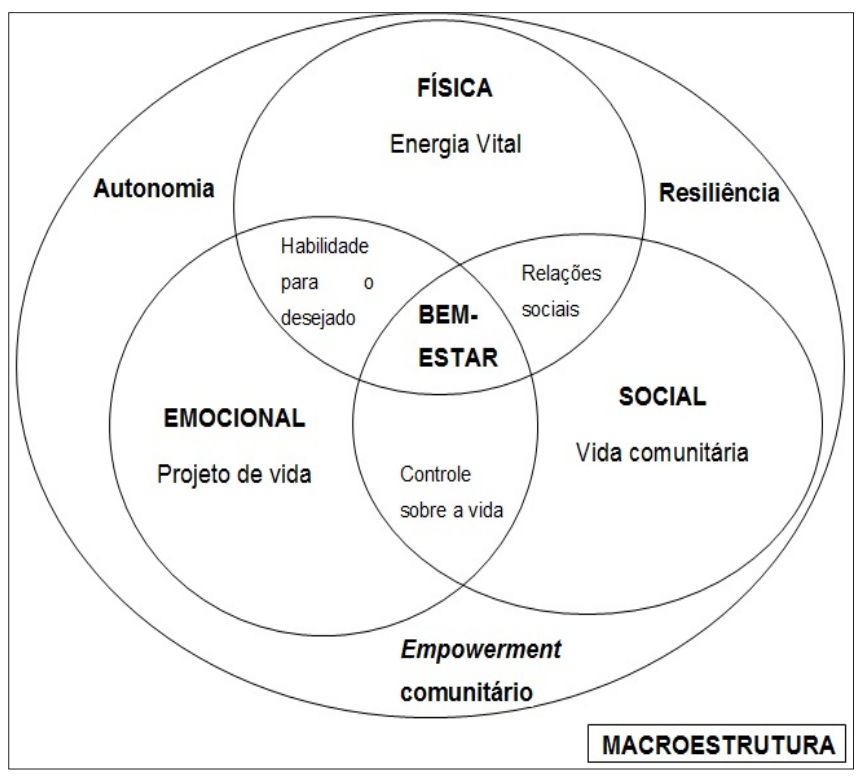

Figura 1. Diagrama de Saúde de Ronald Labonte (1996) e adaptado por Chiesa e colaboradores (2009).

As três dimensões de saúde individual apresentadas por Labonte ${ }^{18}$ são energia vital relacionada ao grau de vitalidade que o corpo biológico dispõe para realizar as atividades da vida diária; projeto de vida representado pelas emoções, aspirações e desejos, que incidem sobre o significado e o sentido de viver projetado pelo indivíduo; vida comunitária expressa pelos relacionamentos e interações do indivíduo em seu entorno, por meio das representações e demais instituições sociais.

As intersecções contidas no Diagrama de Saúde ${ }^{18}$ representam a potencialidade para as intervenções dos profissionais $^{14}$ e são denominadas controle sobre a vida que implica no domínio que o indivíduo tem sobre determinadas condições a fim de desfrutar boas experiências no contexto onde se vive e trabalha; babilidades para realizar o desejado que compreende a tomada de decisões que resultem em realização pessoal e abranjam ações e projetos que atribuem sentido positivo para a vida; e relações sociais que contemplam a construção de redes sociais para o alcance de projetos coletivos e atuação sobre os determinantes sociais de saúde.

Os extratos dos documentos que ilustram as categorias analíticas do referencial teórico adotado ${ }^{18}$ foram codificados em manual de apoio (MJ) e e-book conceitual (BJ) acrescido da paginação original e submetidos à análise lexical por meio do software Interface de $R$ pour les Analyses Multidimensionnelles de Textes et de Questionnaires (IRAMUTEQ), em sua versão 0.7 alpha 2.

$\mathrm{O}$ corpus foi organizado, codificado $\mathrm{e}$ transformado em material adequado ao IRAMUTEQ pelo primeiro pesquisador, especialista em práticas de promoção da saúde, Mestre e Doutor em Ciências com linha de pesquisa em saúde coletiva e educação em saúde. Notas foram tomadas em diário de campo para auxiliar na composição dos temas emergentes em cada dimensão de saúde e bem-estar.

A segunda pesquisadora é Livre-Docente e Doutora na área de enfermagem em saúde coletiva e consultora em projetos voltados à promoção do desenvolvimento infantil. Ressalta-se que ambos os pesquisadores apresentam experiência com investigações qualitativas, são docentes universitários, participaram ativamente do Projeto Janelas e revisaram criticamente os MEI selecionados.

Por se tratar de estudo em base documental, não foi submetido à avaliação de Comitê de Ética em Pesquisa. Entretanto, os princípios éticos recomendados para as atividades de pesquisa em ciências humanas e sociais contidas na resolução 510/2016 foram mantidos durante todas as etapas desta investigação. 


\section{RESULTADOS}

Obtiveram-se 35 temas relacionados ao ideário da promoção da saúde consoante às categorias analíticas (Quadro 1) fundamentadas no Diagrama de Saúde de Ronald Labonte ${ }^{18}$ e que permitem a apreciação da dialética entre a integração de conteúdos biológicos, afetivos e sociais no cuidado infantil proporcionado por diferentes profissionais a fim de incrementar a autonomia crescente das crianças, a resiliência delas e de suas famílias e da sinalização da importância do empoderamento de suas comunidades.

Quadro 1. Caracterização das dimensões de saúde e bem-estar do manual “Toda hora é hora de cuidar”. São Paulo, 2017

\begin{tabular}{|c|c|c|}
\hline Energia vital & Vida comunitária & Projeto de vida \\
\hline $\begin{array}{ll}- & \text { Aleitamento materno; } \\
- & \text { Alimentação nutritiva; } \\
- & \text { Brincadeiras; } \\
- & \text { Desenvolvimento cerebral na infância; } \\
- & \text { Higiene corporal; } \\
- & \text { Imunizações; } \\
- & \text { Prevenção de acidentes e segurança; } \\
- & \text { Problemas comuns na infância; } \\
- & \text { Proteção contra tabagismo, álcool e outras } \\
& \text { drogas; } \\
- & \text { Reconhecimento de deficiências na infância; } \\
- & \text { Risco de automedicação; } \\
- & \text { Saúde bucal; } \\
- & \text { Violência contra a criança e maus tratos. }\end{array}$ & $\begin{array}{ll}\text { - } & \text { Ambiente sustentável; } \\
\text { - } & \text { Estímulos ambientais; } \\
\text { - } & \text { Hospitalização da criança; } \\
\text { - } & \text { Mediação do agente comunitário } \\
& \text { de saúde. }\end{array}$ & $\begin{array}{ll}- & \text { Aprendizagem infantil; } \\
- & \text { Estimulação na primeira } \\
& \text { infância. }\end{array}$ \\
\hline Relações sociais & Habilidade para realizar o desejado & Controle sobre a vida \\
\hline $\begin{array}{ll}\text { - } & \text { Cultura de paz; } \\
- & \text { Garantia de eqüidade; } \\
- & \text { Função materna e paterna; } \\
- & \text { Qualidade dos relacionamentos interpessoais; } \\
- & \text { Patrimônio familiar; } \\
- & \text { Reconhecimento de papéis entre os cuidadores } \\
& \text { da criança. } \\
- & \text { Redes sociais; } \\
- & \text { Relaçóes de solidariedade. }\end{array}$ & $\begin{array}{ll}\text { - } & \text { Ação comunicativa; } \\
\text { - } & \text { Cuidado com enfoque na família; } \\
\text { - } & \text { Diálogo com a criança; } \\
\text { - } & \text { Participação e tomada de decisão na } \\
& \text { infância. }\end{array}$ & $\begin{array}{l}\text { - } \quad \text { Advocacia dos direitos da } \\
\text { criança; } \\
\text { - } \\
\text { - } \quad \text { Invisão de tarefas para o cuidar; } \\
\text { necessidades crianças com } \\
\text { - } \quad \text { Participação e controle social. }\end{array}$ \\
\hline
\end{tabular}

Nas dimensões individuais, houve a predominância da dimensão biológica energia vital em 13 temas (37\%), porém com a incorporação de conteúdos voltados a dois aspectos afetivos dos projetos de vida em dois temas (5\%) e socioambientais da vida comunitária em quatro temas (11\%) para a garantia do desenvolvimento infantil integral.

Nas intersecções que viabilizam a ação profissional, evidenciaram-se oito temas no eixo das relações sociais (22\%) e a coincidência de quatro temas sobre os eixos da habilidade para realizar o desejado (11\%) e do controle sobre a vida (11\%).

Em seguida, com base na terminologia própria da análise lexical do IRAMUTEQ, foram produzidos 36 textos, 155 segmentos de texto, 5.386 ocorrências,
1.736 formas e 1.173 bapax (palavras únicas no corpus). Realizou-se a análise de similitude a fim de reconhecer potenciais conexões entre as palavras mais frequentes nos temas de saúde e bem-estar identificados.

A análise de similitude gerou um gráfico (Figura 2) que possibilitou constatar as relações entre os excertos totais que ilustram as seis dimensões do diagrama de saúde e bem-estar de Ronald Labonte ${ }^{18}$. 


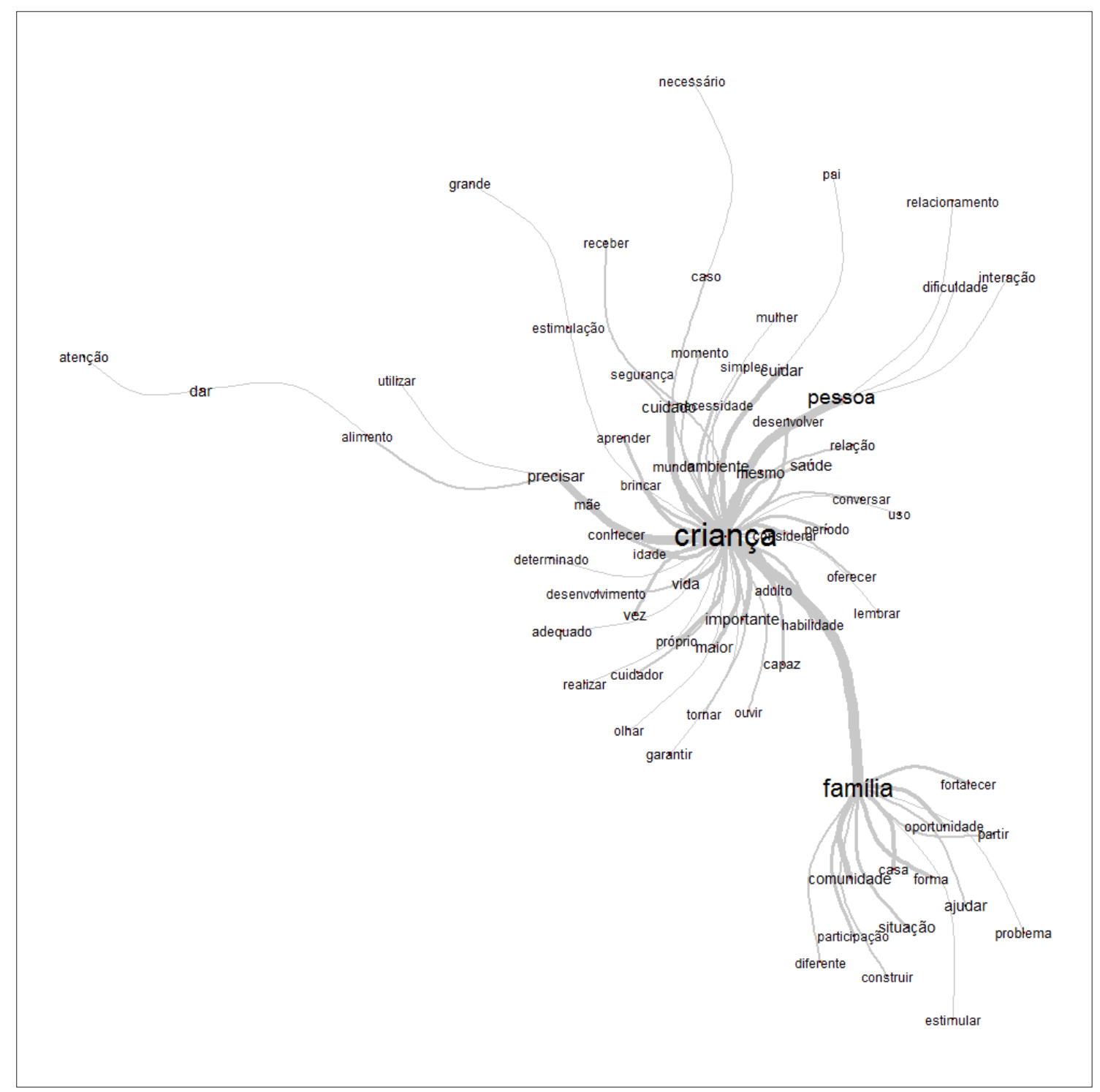

Figura 2. Análise de similitude das dimensões de saúde e bem-estar. São Paulo, 2017.

A figura acima evidencia a importância dada à promoção da saúde da "criança", sem desconsiderar suas famílias nos MEI analisados para fomentar a atuação profissional que prima pela primeira infância. No agrupamento da "criança", com 90 ocorrências textuais, observa-se a valorização das pessoas e suas interações com o intuito de subsidiar práticas centradas nas necessidades em saúde, enquanto que no agrupamento "família", com 52 ocorrências textuais, predomina a noção de fortalecimento das competências para o cuidado realista, participativo e resolutivo da criança pequena.

A seleção de frases e parágrafos pode ajudar na compreensão das dimensões de saúde e bem-estar identificadas no corpus deste estudo. Primeiramente, os extratos referentes à Energia Vital envolveram o cuidado com foco no corpo biológico da criança, desde sua proteção na vida intrauterina até os primeiros seis anos de vida, incluindo os diferentes estímulos das mães, pais ou cuidadores: 
atenção, cuidado". (MJ, p. 72).

"Quando crianças têm oportunidade para estarem na natureza, em ambientes seguros, eles imediatamente saem em campanha de exploração do mundo ao seu redor". (BJ, p. 47 $8)$.

"Caso a família não tenha termômetro, considerar o relato de febre: há muitas pesquisas que mostram que a avaliação pelo tato, realizada por mães, é sensível o suficiente para ser considerada”. (MJ, p. 79).

"[A família] Faz de sua casa um ambiente saudável, evitando drogas, alcoolismo, tabagismo e violência. Ela procura ajuda especializada quando acontece algum desses problemas". (MJ, p. 29).

"O simples escovar dos dentes pode ser aproveitado como um momento de ensinar e compartilhar com o filho este cuidado". (MJ, p. 32).

Em relação à Vida Comunitária, a família da criança e os profissionais de saúde assumem um posicionamento relevante para defender a natureza, o acesso aos serviços e a participação cidadã são representados pelos excertos:

"E todos cuidam de tudo e de todos um pouquinho, construindo um ambiente bonito e saudável...". (MJ, p. 27).

"As habilidades da criança e o seu desempenho são somente relevantes no seu contexto natural. Algumas vezes o ambiente é favorável à criança; outras vezes, não". (BJ, p. 56).

"O trabalho dos agentes comunitários de saúde com as famílias e as comunidades pode ser altamente transformador. É uma contribuição valiosa no sentido de cidadania". (MJ, p. 88).

Os extratos referentes aos Projetos de vida, que devem ser desenvolvidos desde a primeira infância, abordam a importância do brincar e das oportunidades para o desenvolvimento integral, a saber:

"As brincadeiras infantis não são simples passatempos. Através das brincadeiras, as crianças aprendem sobre si mesmas, desenvolvem suas habilidades de movimento, de linguagem, de raciocínio e de interação com o mundo". (MJ, p. 54).
"O desenvolvimento infantil é resultado de uma interação entre forças ambientais e genéticas, com forte predominância das primeiras". (MJ, p. 13).

"O cérebro humano tem uma grande capacidade de desenvolvimento, mas tudo depende do tipo de estimulação e do período em que a mesma acontece". (MJ, p. 13).

"Experiências traumáticas ou ausência de estimulação apropriada prejudicam seriamente o desenvolvimento da criança". (MJ, p. 13).

Para potenciais atuações dos profissionais de saúde nas Relações Sociais, os excertos que ilustram a valorização dos recursos familiares, da vizinhança e ou comunitários para a construção de redes de cuidado fortalecidas foram

"Crianças que têm interações saudáveis contínuas com pessoas que cuidam bem delas tornam-se melhor preparadas, emocional e biologicamente, para aprender e trabalhar estresses e desapontamentos do dia a dia de suas vidas. Isto significa trabalhar diretamente as raízes da violência”. (MJ, p. 10).

"Nossos cuidados e nossa proteção começam antes mesmo do início da gravidez, quando os (futuros) pais cuidam de sua saúde e planejam a família que querem construir". (MJ, p. 24).

"Algumas famílias, mais que outras, dependem muito de apoio externo para dar conta de sua sobrevivência e dos cuidados que seus membros mais jovens necessitam. Estas devem ter prioridade de atenção por parte dos diferentes agentes sociais". (MJ, p. 27).

"Patrimônio indica um conjunto de recursos do qual as pessoas podem dispor para garantir a si mesmas e a seus familiares maior segurança $\mathrm{e}$ melhor padrão de vida. Tais recursos compõem-se de trabalho, saúde, moradia, habilidades pessoais e relacionais - tais como relacionamentos de vizinhança, de amizade, familiares, comunitários e institucionais". (BJ, p. 26).

"A rede social é constituída por um conjunto de relações interpessoais a partir das quais a pessoa e/ou a família mantêm a própria identidade social". (BJ, p. 27). 
"A comunidade, os vizinhos, os parentes e amigos são muito importantes para o fortalecimento da família, especialmente em momentos de crise". (MJ, p. 36).

No tocante à Habilidade para realizar o desejado, a criação de vínculos positivos, a interação comunicativa, a participação da criança em diferentes atividades e a tomada de decisão durante a primeira infância foram representados pelos trechos:

"E que acariciar a criança e conversar, utilizando uma linguagem clara e simples, facilita a construção de um vínculo afetivo positivo e o desenvolvimento da linguagem". (MJ, p. 50).

"Conversar sobre como colocar limites à criança quando necessário, dando explicações simples e sem violência". (MJ, p. 54).

"Todas as pessoas, inclusive as crianças, têm direito e são capazes de escolher o caminho mais apropriado para promover, manter e recuperar sua saúde". (BJ, p. 25).

"Participando das decisões em casa, a criança vai se tornar um adulto seguro e participativo". (MJ, p. 97).

Por fim, no que se refere ao Controle sobre a vida, os excertos que correspondem ao enfrentamento das adversidades, ao empoderamento das famílias e comunidades e ao pleno exercício da cidadania foram ilustrados por:

"Cidadania não é apenas votar e ser votado; é atuar de forma participativa; é respeitar e ser respeitado, ouvir e ser ouvido pela comunidade". (MJ, p. 89).

"Oferecer oportunidades de participação e convivência com todos os membros da família, na vizinhança, na escola e na comunidade. Se a família for ajudada a acessar mais recursos e serviços, isto fortalecerá suas competências na atenção às suas crianças com necessidades especiais". (MJ, p. 59).

"São marcados novos encontros com outros membros da família, vizinhos, parentes, amigos, e, caso seja necessário, com instituições postos de saúde, creches, escolas. É importante conhecer todos os recur- sos da comunidade para poder ajudar a pessoa a usá-los a seu favor". (MJ, p. 44).

Com base na análise temática do conteúdo dos MEI do Projeto Janelas e na complementaridade da análise lexical e de similitude, optou-se por não utilizar um critério de saturação do corpus, sendo que a avaliação das dimensões propostas por Ronald Labonte ${ }^{18}$ e adaptadas por Chiesa e colaboradores ${ }^{14}$ permitiu a ponderação com a literatura nacional e internacional.

\section{DISCUSSÃO}

Os excertos sobre a energia vital traduzem as evidências científicas sobre o desenvolvimento infantil e a citoarquitetura cerebral com bases nos avanços da neurociência e da epigenética ${ }^{20,21}$. Ressalta-se a intencionalidade de superação do modelo biomédico e comportamentalista para um cuidado integral e integrado na primeira infância para a reorientação das práticas profissionais a fim de oferecer suporte às crianças, suas famílias e comunidades ${ }^{22-25}$.

Apesar da predominância de temas relacionados ao crescimento biológico e aos comportamentos observáveis na infância, o manual de apoio incluiu mensagens que abordam transversalmente as dimensões da vida comunitária da família de crianças de zero a seis anos e suas interfaces com a vizinhança e demais recursos comunitários $^{14,23-26}$.

A construção do projeto de vida da criança mediado por seus cuidadores também foi considerado ao abarcar a cognição, o desejo, o afeto, os sentimentos presentes em todo o desenvolvimento humano e que influenciam a construção de valores, posturas e modos de levar a vida ${ }^{21-24}$

No tocante às possibilidades de intervenção, as intersecções apresentadas pelo diagrama de Labonte ${ }^{18}$ proporcionam um espaço para o desenvolvimento de tecnologias de cuidado e abordagens de intervenção junto à família para promover a saúde e o desenvolvimento infantil de forma mais integral, equitativa e efetiva ${ }^{14}$.

No eixo das relações sociais, o corpus do presente estudo revela a importância da construção de 
uma rede de cuidados interprofissionais e intersetoriais para o fortalecimento das competências dos familiares e dos cuidadores das crianças de zero a seis anos a $^{21,22,25,26}$.

A autonomia, a liberdade, a criatividade e a autorrealização da criança, dentro de um plano de aspirações individuais compartilhados no âmbito familiar, foram identificados em extratos que atribuem significados positivos para o eixo do desenvolvimento da habilidade para realizar o desejado $0^{22-24,26}$.

Para o eixo do controle sobre a vida, as mensagens destacadas do corpus analisado evidenciaram a defesa dos direitos de cidadania, o controle social, a avaliação de necessidades específicas para condições limitantes e o mapeamento dos relacionamentos familiares e de diferentes atores sociais que protagonizam o cuidar e o educar na primeira infância ${ }^{22-26}$.

Os temas relacionados à energia vital contemplaram a fisiologia do corpo biológico (avanços da neurociência e da epigenética) e os cuidados necessários para mantê-lo saudável (alimentação nutritiva, higiene corporal, imunizações, saúde bucal, prevenção de acidentes e segurança, proteção contra tabagismo, álcool e outras drogas).

Identificou-se a transversalidade de temas que superam a abordagem tradicional de temas de caráter prescritivo e curativo na atenção à saúde da criança e que implicam na defesa dos recursos e patrimônio da família (alimentação nutritiva, problemas comuns na infância, risco de automedicação), o fortalecimento do contexto e das relações interpessoais estabelecidas entre os cuidadores e a criança (aleitamento materno, amamentação, brincadeiras), além da possibilidade de identificar abusos contra a criança (violência contra a criança e maus tratos) e processos doentios e/ou degenerativos (reconhecimento de deficiências na infância).

Os aspectos relacionados à vida comunitária foram representados pela construção de hábitos que zelem pelo entorno social, desenvolvam habilidades, estimulem o aprendizado e cultivem um sentimento de pertença cultural (ambientes saudáveis, estimulantes e seguros). As oportunidades de acesso aos serviços de saúde desde a atenção primária (mediação do agente comunitário de saúde e uso dos instrumentos do Projeto Janelas) até a atenção secundária e terciária (hospitalização da criança) foram discutidas para evitar mudanças na rotina, sofrimentos e gastos desnecessários para a família.

Por se tratar de um corpus direcionado para a primeira infância, os conteúdos pertencentes ao projeto de vida da criança ficaram limitados à consolidação de atitudes e cuidados (aprendizagem infantil e estimulação na primeira infância) a fim de favorecer um desenvolvimento infantil adequado e uma vida adulta mais produtiva e saudável que contemple sua autorrealização e o enfrentamento positivo de problemas.

As relações sociais concentraram-se no espaço familiar e expressaram os relacionamentos da criança com seus cuidadores (função materna e paterna, qualidade dos relacionamentos interpessoais e reconhecimento de papéis entre os cuidadores da criança), assim como sua estrutura para garantir suas necessidades (patrimônio familiar). No entanto, o apoio fornecido por familiares e pela vizinhança (cultura de paz e relações de solidariedade) e pelos membros da comunidade ou profissionais de diversas áreas de atuação (redes sociais) foi associado a outros temas.

Entende-se que o manual de apoio e o e-book conceitual do Projeto Janelas demonstrou a consolidação dos princípios norteadores da promoção da saúde para que os profissionais envolvidos na produção de cuidados integrais e integrados a crianças de zero a seis anos, com ênfase nos três primeiros anos de vida, possam incorporar conteúdos inovadores e temas relevantes a suas práticas e seus processos de trabalho ${ }^{27}$.

A produção, difusão e utilização do conhecimento devem considerar a complexidade dos processos de incorporação de tecnologias de cuidado, pois o setor saúde apresenta uma interface sui generis entre o bem-estar e a adoção de inovações científicas que deve ser considerado na elaboração de instrumentos concebidos para apoiar as práticas educativas em saúde transformadoras ${ }^{28}$.

Outra possibilidade que pode favorecer a formação contínua dos profissionais compreende a utilização de acervos digitais que tornam os MEI disponíveis em versões eletrônicas e podem favorecer a translação do conhecimento, ou seja, um processo que visa 
à redução da lacuna entre o conhecimento produzido em instituições de ensino e de pesquisa e sua aplicabilidade pelos atores sociais relevantes dos diferentes segmentos da sociedade em prol da atual e futura geração $0^{29}$, assim como no fortalecimento das estratégias de educação permanente no Sistema Único de Saúde ${ }^{30}$.

\section{CONCLUSÃO}

A construção interprofissional e intersetorial de tecnologias inovadoras para a promoção da saúde na primeira infância derivadas do projeto "Nossas crianças: janelas de oportunidades" demonstra a potencialidade da elaboração colaborativa do manual "Toda hora é hora de cuidar" e do e-book conceitual para a educação permanente de profissionais envolvidos com o cuidado de gestantes e de crianças de zero a seis anos.

Entende-se que analisar dois documentos de um caso exemplar representa a limitação do presente estudo. Por essa razão, optou-se pela análise lexical e conceitual em profundidade com a intenção de apresentar uma estratégia de avaliação de conteúdos de saúde e bemestar presentes em materiais educativos analógicos $\mathrm{e}$ ou digitais a fim de subsidiar o exercício das equipes de saúde na garantia da integralidade do cuidado infantil, familiar e comunitário.

O diagrama de saúde e bem-estar, proposto por Ronald Labonte, pode subsidiar análises de MEI que enfatizam a promoção da saúde a fim de transcender a abordagem biomédica (centrada na doença) e comportamentalista (com ênfase nos fatores de risco e estilos de vida) em práticas educativas em saúde, pois incorpora a positividade desses modelos prévios e avança na incorporação transversal de aspectos afetivos, culturais e socioambientais inerentes ao processo saúdedoença e à produção de cuidados individuais, familiares e coletivos.

Compreender a fundamentação conceitual e metodológica do paradigma da promoção da saúde pode incrementar as dimensões técnica, ética, política e estética contidas nos MEI e propiciar uma avaliação crítica e epistemológica de recursos instrucionais potencialmente significativos para promover mudanças positivas na saúde, na qualidade de vida e bem-estar desde a vida intrauterina.

\section{REFERÊNCIAS}

1. Giguère A, Légaré F, Grimshaw J, Turcotte $S$, Fiander M, Grudniewicz A et al. Printed educational materials: effects on professional practice and healthcare outcomes. Cochrane Database Syst Rev. 2012(10). DOI: 10.1002/14651858.CD004398.pub3.

2. Canada. The Centre for Literacy. The Calgary Charter on Health Literacy: rationale and core principles for the development of health literacy curricula [Internet]. Montreal; 2011 [cited 2018 Jan. 27]. Available from: http://www.centreforliteracy.qc.ca/ sites/default/files/CFL_Calgary_Charter_2011.pdf.

3. Grippo MLVS, Fracolli LA. Evaluation of an educational booklet about childcare promotion from the family's perception regarding health and citizenship. Rev. Esc. Enferm. USP [Internet]. 2008 [cited 2018 Jan. 27];42(3):430-6. Available from: http://www.scielo. br/pdf/reeusp/v42n3/en_v42n3a02.pdf.

4. Martins J, Veríssimo MDLOR, Oliveira MA. Health agents' evaluation of the project instruments in: "our children - windows of opportunities". Texto Contexto Enferm. [Internet]. 2008 [cited 2018 Jan. 27];17(1):106-14. Available from: http://www.scielo. $\mathrm{br} / \mathrm{pdf} / \mathrm{tce} / \mathrm{v} 17 \mathrm{n} 1 / 12$.pdf.

5. Reberte LM, Hoga LAK, Gomes ALZ. Process of construction of an educational booklet for health promotion of pregnant women. Rev. Latino-Am. Enfermagem [Internet]. 2012 [cited 2018 Jan. 27];20(1):101-8. Available from: http://www.scielo. br/pdf/rlae/v20n1/pt 14 .

6. Levis DM, Westbrook K. A content analysis of preconception health education materials: characteristics, strategies, and clinical-behavioral components. Am. J. Health Promot. [Internet]. 2013 [cited 2018 Jan. 27];Suppl 3:S36-42. Available from: http://ajhpcontents.org/doi/pdf/10.4278/ ajhp.120113-QUAL-19.

7. Zombini EV, Pelicioni MCF. Strategies for evaluation of an educational material in eye health. Rev. Bras. Cresc. Desenv. Hum. [Internet]. 2011 [cited 2018 Jan. 27];21(1):51-8. Available from: http://pepsic. bvsalud.org/pdf/rbcdh/v21n1/06.pdf. 
8. Torres HC, Candido NA, Alexandre LR, Pereira FL. The process of creating guidebooks for orienting self-care in the diabetes educational program. Rev. Bras. Enferm. [Internet]. 2009 [cited 2018 Jan. 27];62(2):312-6. Available from: http://www.scielo. br/pdf/reben/v62n2/a23v62n2.pdf.

9. Gozzo TO, Lopes RR, Prado MAS, Cruz LAP, Almeida AM. Information to the development of an educational manual for women with breast cancer. Esc. Anna Nery [Internet]. 2012 [cited 2018 Jan. 27];16(2):306-11. Available from: http://www.scielo. br/pdf/ean/v16n2/14.pdf.

10. Coriolano-Marinus MWL, Pavan MI, Lima LS, Bettencourt ARC. Validation of educational material for hospital discharge of patients with prolonged domiciliary oxygen prescription. Esc. Anna Nery [Internet]. 2014 [cited 2018 Jan. 27];18(2):284-9. Available from: http://www.scielo.br/pdf/ean/v18n2/ en_1414-8145-ean-18-02-0284.pdf.

11. Barrera Sánchez LF, Manrique Abril FG, Ospina Díaz JM. Propiedades psicométricas de instrumentos utilizados para evaluar material educativo en salud. Lina F. Hacia la Promoción de la Salud [Internet]. 2011 [cited 2018 Jan. 27];16(1):13-26. Available from: http://promocionsalud.ucaldas.edu.co/downloads/ Revista16\%281\%29_2.pdf.

12. Castro MS, Pilger D, Fuchs FD, Ferreira MBC. Development and validity of a method for the evaluation of printed education material. Pharmacy Practice [Internet]. 2007 [cited 2018 Jan. 27] ; 5(2):8994. Available from: http://scielo.isciii.es/pdf/ pharmacin/v5n2/089-094.pdf.

13. Nespoli G, Ribeiro VMB. Discourses that form knowledge: an analysis of the theoretical and methodological conceptions that guide the educational material for the training of facilitators of Continuing Education in Health. Interface (Botucatu) [Internet]. 2011 [cited 2018 Jan. 27]; 15(39):985-96. Available from: http://www.scielo.br/pdf/icse/v15n39/ aop2911.pdf.
14. Chiesa AM, Fracolli LA, Verissimo MDLO, Zoboli ELCP, Ávila LK, Oliveira AAP. Building health care technologies based on health promotion. Rev. Esc. Enferm. USP [Internet]. 2009 [cited 2018 Jan. 27];43(2):1352-7. Available from: http://www.scielo. br/pdf/reeusp/v43nspe2/en_a36v43s2.pdf.

15. Zoboli ELCP, Fracolli LA, Chiesa AM. Nossas crianças: toda hora é hora de cuidar. Revista de Cultura e Extensão USP [Internet]. 2010 [cited 2018 Jan. 27];4:17-24. Available from: http://www.revistas.usp. br/rce/article/view/478/477.

16. Chiesa AM, Fracolli LA. A percepção das famílias sobre a cartilha "toda hora é hora de cuidar". O Mundo da Saúde [Internet]. 2010 [cited 2018 Jan. 27];34(1):3642. Available from: http://www.saocamilo-sp.br/pdf/ mundo_saude/74/04_original_Percepcao.pdf.

17. Minayo MCS. O desafio do conhecimento. Pesquisa qualitativa em saúde. 14. ed. São Paulo; 2014.

18. Labonte R. Health promotion and empowerment: practice frameworks. Toronto (Canada): Center for Health Promotion at University of Toronto; 1996.

19. Camargo BV, Justo AM. IRAMUTEQ: um software gratuito para análise de dados textuais. Temas Psicol. [Internet]. 2013 [cited 2018 Jan. 27];21(2): 513-8. Available from: http://pepsic.bvsalud. org/scielo.php?script $=$ sci_arttext $\&$ pid $=$ S1413389X2013000200016\&lng $=$ pt .

20. Shonkoff JA, Bales WN. Science does not speak for itself: translating child development research for the public and its policymakers. Child Dev. [Internet]. 2011 [cited 2018 Jan. 27];82(1):1732. Available from: http://eadershiplinc. illinoisstate.edu/researchcompendium/documents/ ScienceDoesNotSpeakforItself.pdf.

21. Crews D, Gillette R, Miller-Crews I, Gore AC, Skinner MK. Nature, nurture and Epigenetics. Molecular and cellular endocrinology [Internet]. 2014[cited 2018 Jan. 27];398(0):42-52. Available from: https://www. ncbi.nlm.nih.gov/pmc/articles/PMC4300943/pdf/ nihms619154.pdf.

22. Cypel S, organizador. Fundamentos do 
desenvolvimento infantil: da gestação aos 3 anos [Internet]. São Paulo: Fundação Maria Cecília Souto Vidigal; 2011 [cited 2018 Jan. 27]. Available from: http://www.fmcsv.org.br/pt-br/acervo-digital/Paginas/ Fundamentos-do-desenvolvimento-infantil.aspx.

23. Yousafzai AK, RasheedMA, Daelmans B, Manji S, Arnold C, Lingam R, et al. Capacity building in the health sector to improve care for child nutrition and development. Ann. N.Y. Acad. Sci. [Internet].2014 [cited 2018 Jan. 27];1308:172-82. Available from: http://onlinelibrary.wiley.com/doi/10.1111/ nyas.12351/epdf.

24. Souza JM, Veríssimo MLOR. Desenvolvimento infantil: análise de um novo conceito. Rev. LatinoAm. Enfermagem [Internet]. 2015 [cited 2018 Jan. 27];23(6):1097-1104. Available from: https://www. revistas.usp.br/rlae/article/view/108021.

25. Woolfenden S, Goldfeld S, Shanti R, Kemp L, Williams $\mathrm{K}$. Inequity in child health: the importance of early childhood development. J. Paediatr. Child Health. 2013;49(9):365-9.

26. Rede Nacional pela Primeira Infância. Plano Nacional pela Primeira Infância [Internet]. Brasília; 2010 [cited 2018 Jan. 27]. Available from: http://primeirainfancia. org.br/wp-content/uploads/2015/01/PNPI-Resumido. pdf.

27. Oliveira AAP, Moreira RL, Pécora RAF, Chiesa AM. Relevant themes for child development training: a case study in light of health promotion. Revista Medicina (São Paulo) [Internet]. 2013 [cited 2018Jan. 27];92(2):113-8. Available from: http://www.revistas. usp.br/revistadc/article/viewFile/79949/83884.

28. Miranda MCG, Almeida BA, Aragão E, Guimarães JM. Política nacional de ciência, tecnologia e inovação em saúde e a necessidade de educação permanente. Rev. Baiana de Saúde Pública [Internet]. 2012 [cited 2018 Jan. 27];36(1):82-9. Available from: http://inseer.ibict. br/rbsp/index.php/rbsp/article/viewFile/238/pdf 53.

29. Pina-Oliveira AA, Germani ACCG, Chiesa AM. Potentialities and limitations of a digital repository on a programme to promote the early childhood development. Rev. Eletrônica Comun., Inf. Inov. Saúde [Internet]. 2014 [cited 2018 Jan. 27];8(3):41324. Available from: http://www.reciis.icict.fiocruz.br/ index.php/reciis/article/view/688/1335.

30. Silva JN, Rodrigues VD, Silva AG. Educação permanente em saúde através da educação à distância: uma breve introdução. Revista Saúde e Pesquisa [Internet]. 2013 [cited 2018 Jan. 27];6(3):503-9. Available from: http://periodicos.unicesumar.edu.br/ index.php/saudpesq/article/view/3033/2163.

Recebido em: 04/01/2018 Aceito em: 25/05/2018 